\title{
Nanoantibiotics: strategic assets in the fight against drug-resistant superbugs
}

This article was published in the following Dove Press journal:

International Journal of Nanomedicine

\section{Chandni Khurana \\ Bhupendra Chudasama}

Laboratory of Nanomedicine, School of Physics and Materials Science, Thapar University, Patiala, Punjab, India
Correspondence: Bhupendra Chudasama Laboratory of Nanomedicine, School of Physics and Materials Science, Thapar University, Patiala, Punjab 147004, India Email bnchudasama@gmail.com

\begin{abstract}
Antimicrobial characteristics of metals reveal that Ag despite its economic constraints remains the most popular antibiotic agent. Antimicrobial characteristics of copper nanoparticles (CNPs) are not well understood. To our knowledge, no systematic comparative study on microbial properties of silver nanoparticles (SNPs) and CNPs exists. In this article, a comparative study on microbial properties of engineered metal nanoantibiotics against clinically important strains has been attempted. Our results indicate that biocidal activities of CNPs are better than SNPs. Minimum inhibitory concentration (MIC) values of CNPs are 10 times lower than the corresponding MICs of SNPs. These improved biocidal activities of CNPs would make it affordable and potent nontraditional antibiotics against which microbes are least susceptible to develop any drug resistance.
\end{abstract}

Keywords: antibiotics, silver, copper, nanoparticles

\section{Introduction}

Infectious diseases continue to be one of the greatest health challenges worldwide. Antibiotic resistance in microbes is becoming increasingly important in public health care due to recent breakouts of microbial resistance. The World Health Organisation report in 2014 on global surveillance of antimicrobial resistance reveals that antibiotic resistance is putting at risk the ability to treat common infections. ${ }^{1}$ The world is heading toward a postantibiotic era. Excessive and uncontrolled use of antibiotics, both in preventive and curative health care, has worsened the situation, as microbes, which have never been exposed to an antibiotic are acquiring resistance toward that drug due to transfer of gene coding. ${ }^{2}$ Hence, fighting multidrug resistance with traditional antibiotics is getting tougher and need is being felt for the development of nontraditional antibiotics against which microbes are unlikely to acquire resistance. ${ }^{3}$

Superbugs are unlikely to develop resistance against metal nanostructures as they commonly do against conventional antibiotics because of their broad and random targeting capabilities. Existing literature on antimicrobial characteristics of metals reveals that $\mathrm{Ag}$ despite its economic constraints remains to be the most popular antibiotic agent. A low-cost alternative to silver nanoparticles (SNPs) could be copper nanoparticles (CNPs). Antimicrobial properties of CNPs are in infancy. To our knowledge, no systematic comparative study on microbial properties of SNPs and CNPs exists. In this article, we report about the comparative study on microbial properties of engineered metal nanoantibiotics against clinically important strains. 


\section{Materials and methods}

\section{Materials}

Antibacterial activities have been investigated on clinically important strains of Escherichia coli (MTCC No 77), Staphylococcus aureus (MTCC No 9760), and Proteus vulgaris (MTCC No 426). Mueller-Hinton agar and nutrient broth were used to prepare growth media.

\section{Synthesis of SNPs and CNPs}

In this study, synthesis of uniform, monodisperse SNPs is carried out by simple one-pot method using oleylamine (OA) as a reducing and capping agent. ${ }^{4}$ It is a two-step process. In the first step, OA-capped hydrophobic SNPs are prepared by reducing $\mathrm{AgNO}_{3}$ with OA. In the second step, hydrophobic SNPs are phase transferred into water by ligand exchange reaction using pluronic F-127. Colloidal dispersion of SNPs is used as nanoantibiotics-I. CNPs are synthesized by the chemical reduction of $\mathrm{CuCl}_{2} \cdot 2 \mathrm{H}_{2} \mathrm{O}$ under mild reaction conditions by using a unique combination of reducing (sodium borohydride and L-ascorbic acid) and capping (polyvinylpyrrolidone and L-ascorbic acid) agents. Synthesized CNPs are stable in its native metallic state $\left(\mathrm{Cu}^{0}\right)$ under the dynamic equilibrium established between the surface of CNPs and the capping agents, which is called nanoantibiotics-II in this study.

\section{Antibacterial activity}

Antibacterial activities of nanoantibiotics were investigated by microdilution and disk diffusion tests. In the microdilution test, nutrient broth medium containing nanoantibiotics with effective concentration between 0 and $150 \mu \mathrm{g} / \mathrm{mL}$ were inoculated with $10^{8} \mathrm{CFU} / \mathrm{mL}$ of the respective bacterial suspension. The inoculated sets were incubated at $37^{\circ} \mathrm{C}$ for $24 \mathrm{~h}$. Lowest concentration of nanoantibiotics that inhibited bacterial growth was taken as minimum inhibitory concentration (MIC) for that particular bacterium. ${ }^{5}$ Biocidal activities of nanoantibiotics were also investigated by disk diffusion test. Mueller-Hinton agar was added to disposable petridishes and allowed to solidify. Each bacterial strain was inoculated onto the entire surface of Mueller-Hinton agar plates. A sample disk containing appropriate quantity of nanoantibiotic was placed at the center of the disk and incubated at $37^{\circ} \mathrm{C}$ for $24 \mathrm{~h}$. The zone of inhibition was measured as the area on the agar plate where the colony formation was inhibited. ${ }^{6}$

\section{Results and discussion}

Engineered nanostructures of SNPs and CNPs are characterized by X-ray diffraction and transmission electron microscopy (Figure 1). Diffraction study reveals that both nanoparticles crystallize into face-centered cubic unit cells. No impurity or oxide phases are observed in the diffractograms. Transmission electron microscopy micrographs reveal that both SNPs and CNPs are monodisperse with physical sizes of 8 and $2 \mathrm{~nm}$, respectively. Self-assembly of nanostructures indicates that they are highly stable in colloidal state. Results of biocidal activities of nanoantibiotics are summarized in Table 1.

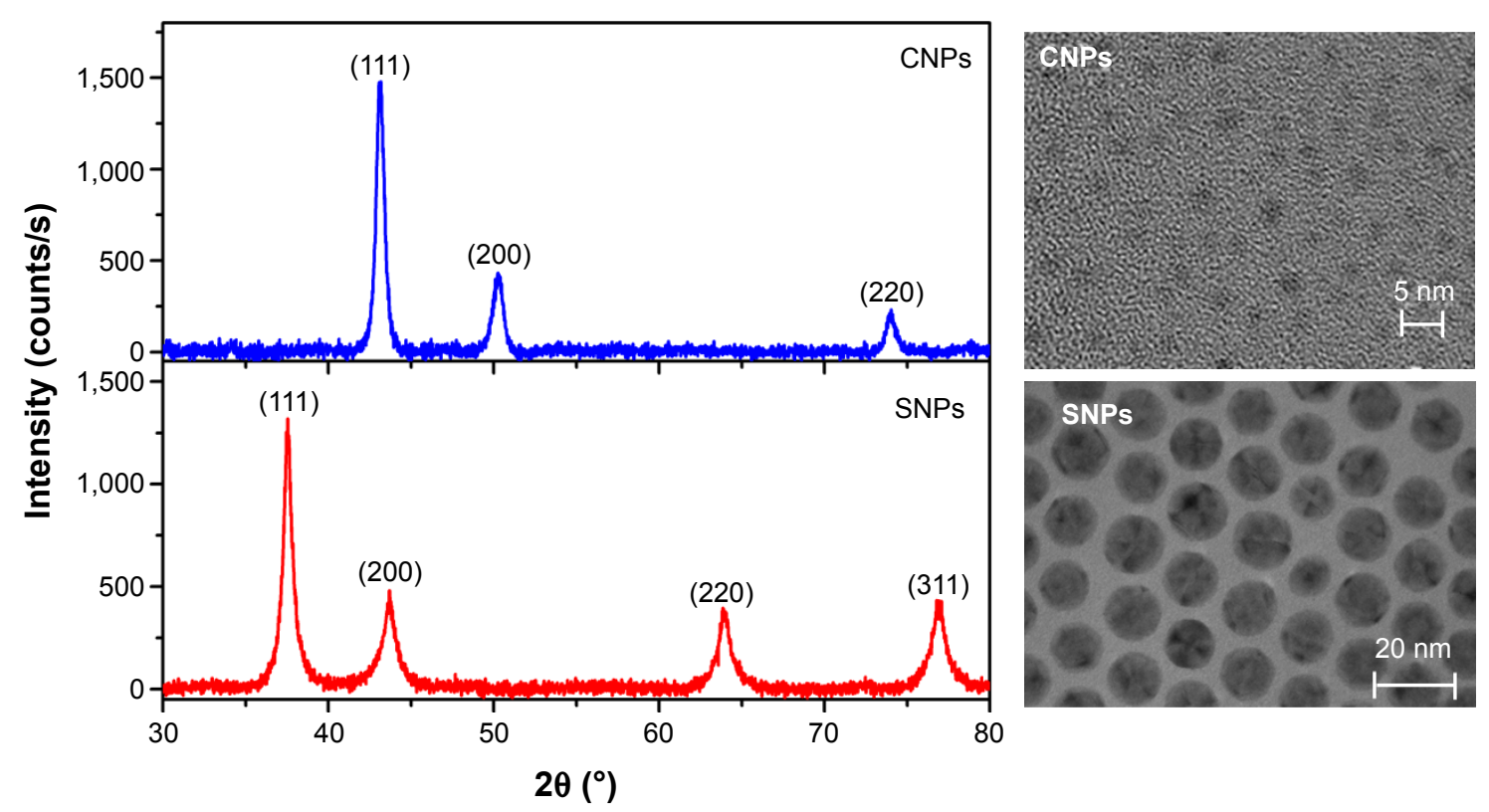

Figure I X-ray diffractograms and TEM images of engineered nanostructures of SNPs and CNPs.

Abbreviations: CNPs, copper nanoparticles; SNPs, silver nanoparticles; TEM, transmission electron microscopy. 
Table I MIC and ZIH data of nanoantibiotics-I and nanoantibiotics-II against clinically important pathogens

\begin{tabular}{|c|c|c|c|c|}
\hline \multirow[t]{2}{*}{ Strains } & \multicolumn{2}{|l|}{ MIC ( $\mu \mathrm{g} / \mathrm{mL})$} & \multicolumn{2}{|l|}{ ZIH (mm) } \\
\hline & Nanoantibiotic-I & Nanoantibiotic-II & Nanoantibiotic-I & Nanoantibiotic-II \\
\hline Escherichia coli & $30 \pm 5$ & $20 \pm 5$ & $12 \pm 0.1$ & $23 \pm 0.1$ \\
\hline Staphylococcus aureus & $150 \pm 5$ & $20 \pm 5$ & $I I \pm 0.1$ & $18 \pm 0.2$ \\
\hline Proteus vulgaris & $100 \pm 5$ & $30 \pm 5$ & $7 \pm 0.2$ & $28 \pm 0.2$ \\
\hline
\end{tabular}

Note: Data presented as mean \pm SD.

Abbreviations: MIC, minimum inhibitory concentration; $\mathrm{ZIH}$, zone of inhibition.

E. coli is most susceptible to nanoantibiotics followed by $P$. vulgaris and $S$. aureus. Results of disk diffusion test are shown in Figure 2. Among antibiotics, nanoantibiotics-II is more potent to all tested microorganisms. Observed MIC values of nanoantibiotics are low and comparable to those reported for commercial antibiotics. CNPs-based nanoantibiotics-II would be a better and cost-effective alternative to narrow targeting commercial antibiotics. These nanoantibiotics are unlikely to experience any drug resistance due to their broad and random targeting capabilities. These observed high biocidal activities of engineered nanoantibiotics could be ascribed to their high colloidal stability and capabilities to resist surface oxidation.
Poisoning of microbial cells by nanoantibiotics is a multimodal phenomenon. This includes, but not limited to impaired membrane function, generation of reactive oxygen species and antioxidant depletion, protein dysfunction, genotoxicity, interference with nutrient assimilation and so on.

\section{Conclusion}

Biocidal activities of CNPs are better than SNPs. MIC values of CNPs are 10 times lower than the corresponding MICs of SNPs. Improved biocidal activities of CNPs would stem further efforts in the quest for affordable and potent nontraditional antibiotics against which microbes are least susceptible to develop any drug resistance.
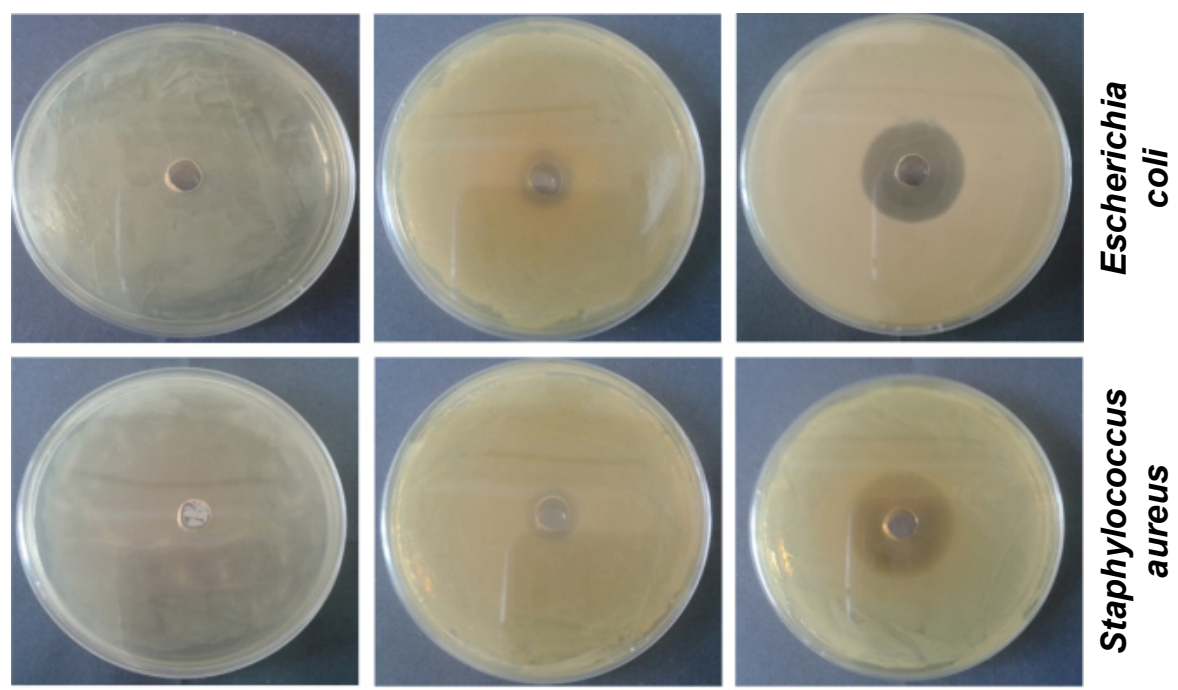

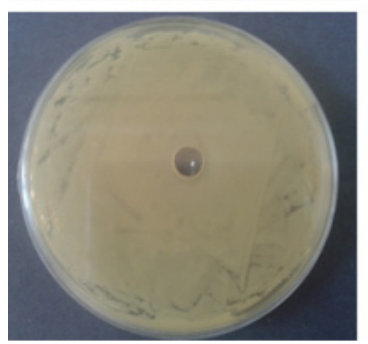

Control

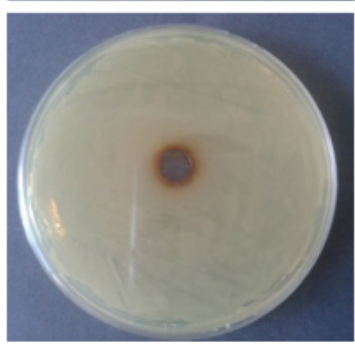

Nanoantibiotics-I

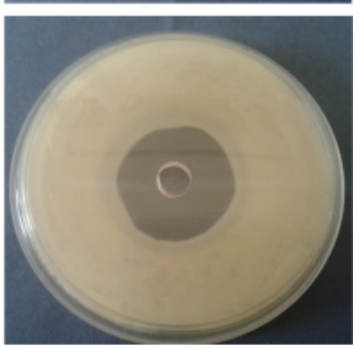

Nanoantibiotics-II

Figure 2 Photographic view of $\mathrm{ZIH}$.

Note: Control represents microbial growth in the absence of nanoantibiotics.

Abbreviation: $\mathrm{ZIH}$, zone of inhibition. 


\section{Acknowledgment}

The authors acknowledge Council for Scientific and Industrial Research and University Grants Commission, India for financial support.

\section{Disclosure}

The authors report no conflicts of interest in this work.

\section{References}

1. World Health Organization. Antimicrobial Resistance Global Report on Surveillance, World Health Organization (2014). Available from: http://www.who.int/drugresistance/documents/surveillancereport/en/. Accessed December 8, 2016.
2. Hopkins DR. The Greatest Killer: Smallpox in History. 1st ed. Chicago, IL: The University of Chicago Press; 2002.

3. Kon K, Rai M. Metallic nanoparticles: mechanism of antibacterial action and influencing factors. J Comp Clin Path Res. 2013;2(1):160-174.

4. Chandni, Andhariya N, Pandey OP, Chudasama B. A growth kinetics study of ultrafine monodispersed silver nanoparticles. RSC Adv. 2013;3(4): $1127-1136$.

5. Khurana C, Vala AK, Andhariya N, Pandey OP, Chudasama B. Antibacterial activity of silver: the role of hydrodynamic particle size at nanoscale. J Biomed Mater Res A. 2014;102(10):3361-3368.

6. Khurana C, Vala AK, Andhariya N, Pandey OP, Chudasama B. Antibacterial activities of silver nanoparticles and antibiotics adsorbed silver nanoparticles against biorecycling microbes. Environ Sci Processes Impacts. 2014;16(9):2191-2198.

\section{Publish your work in this journal}

The International Journal of Nanomedicine is an international, peerreviewed journal focusing on the application of nanotechnology in diagnostics, therapeutics, and drug delivery systems throughout the biomedical field. This journal is indexed on PubMed Central, MedLine, CAS, SciSearch ${ }^{\circledR}$, Current Contents ${ }^{\circledR} /$ Clinical Medicine,

Journal Citation Reports/Science Edition, EMBase, Scopus and the Elsevier Bibliographic databases. The manuscript management system is completely online and includes a very quick and fair peer-review system, which is all easy to use. Visit http://www.dovepress.com/ testimonials.php to read real quotes from published authors. 